\title{
Circular Cryogenic Excision in Surgical Treatment of Skin Melanoma
}

DOI: 10.17691/stm2018.10.3.6

Received October 8, 2017

T.G. Kotova, MD, PhD, Researcher".

V.1. Kochenov, MD, DSc, Senior Researcher, Department of Operative Surgery

and Topographic Anatomy²; Director ${ }^{1}$;

T.E. Potemina, MD, DSC, Professor, Head of the Department of Pathophysiology2;

S.N. Tsybusov, MD, DSC, Professor, Head of the Department of Operative Surgery and Topographic Anatomy ${ }^{2}$

I"Oncolor" Scientific Clinical Center for Cryo-Medicine, 4a Beketova St., Nizhny Novgorod, 603057, Russia;

2Privolzhsky Research Medical University, 10/1 Minin and Pozharsky Square, Nizhny Novgorod, 603005, Russia

The aim of the study was to evaluate the efficacy of circular cryo-excision in the surgical treatment of skin melanomas.

Materials and Methods. The study involved 126 patients with histologically verified malignant skin melanomas. The treatment efficacy was assessed by measuring the levels of antibodies to the CD3, CD16, CD4, and CD8 lymphocytes followed by calculating the CD4/CD8 ratio (immune regulation index, IRI) as an index of the immune health. For deep freezing of skin melanoma, a continuously cooled applicator with a ring-shaped tip attached to a vertical tube was used. Cryosurgical intervention - an excision of tumor tissues - was carried out in three stages.

Results. The CD4/CD8 ratio (IRI) before the cryosurgery was $0.6 \pm 0.1$; after the treatment, the IRI increased 2-fold. In 6 months, the level of IRI reached 1.5 \pm 0.2 . The 5 -year survival rates after the circular cryo-excision were $80.0 \pm 8.9 \%$, even for melanomas with the Clark index of IV-V.

Conclusion. Cryosurgical treatment of melanoma has a stimulating effect on the immune system and causes therapeutic consolidation that lasts for about 48 months, depending on the reactivity of the immune system. The results demonstrate the efficacy of this novel approach and rationalize its further use in oncosurgery.

Key words: circular cryo-excision; cryosurgery of melanoma; cryotherapy of melanoma; skin melanoma; skin cancer; immune status.

\section{Introduction}

Melanoma is one of the most malignant and aggressive skin formations with rapid progression and poor prognosis; the disease accounts for 1.0 to $4.0 \%$ of all cancers [1, 2]. The number of people suffering from melanoma is growing rapidly every year. Thus, in the USA, the incidence of skin melanoma increases by $4.0 \%$ annually, and in Russia this figure reaches $4.95 \%$ [3].

Back in the 1990s, it became obvious that thermoablative technologies had a number of advantages over traditional methods of surgical treatment of tumors: ablastic manipulations; reduced area of tissue removal; fewer complications, cost and time effectiveness; an extended range of indications as compared with traditional surgery [4]. It is also important that cryogenic treatment does not require anesthesia and that the surgical procedure is less complicated than traditional melanoma surgery [5, 6]. In addition, the use of low and ultralow temperatures makes it possible to activate the oncocytolytic mechanism and strengthen the therapeutic efficacy of such antitumor drugs as mitomycin [7, 8]. The oncocytolytic effect involves a spread of tumor antigens as a result of cryo-exposure; those antigens stimulate the production of cytotoxic $T$ lymphocytes capable of attacking tumor cells $[4,9,10]$.

The specific freeze-thaw cycles allow for directly killing tumor cells, triggering apoptosis, and also inhibiting the growth of surviving cells [11]. Another evidence of the pronounced immunological effect of cryo-exposure came from a study on treatment of skin metastases of melanoma using cryotherapy combined with Imiquimod (cytokine inducer) $[12,13]$.

Among the known methods of cryosurgery, the best studied is the one used in the treatment of malignant lentigo [14-17]. This approach became a method of choice for the management of elderly patients and patients with contraindications against traditional surgery or general anesthesia.

The aim of the study was to evaluate the efficacy of circular cryo-excision in the surgical treatment of skin melanomas by using the immunological indices and the 5 -year survival data. 


\section{Materials and Methods}

The study was conducted at the "Oncolor" Scientific Clinical Center for Cryo-Medicine (Russia). There were 126 patients with confirmed diagnosis of "malignant skin melanoma" without clinical manifestations of metastasis, including 49 men $(38.9 \%)$ and 77 women $(61.1 \%)$. The average age of the patients was $63.6 \pm 0.8$ years. By melanoma location, they were grouped as follows: torso - 31\% (39/126); upper and lower limbs - 43.7\% (55/126); head and neck - 25.3\% (32/126). According to the Clark invasion depth index, the number of patients with grades I-II were 81 , with grade III -20 , and with grades IV-V degrees -25 patients.

The study was conducted in accordance with the rules and regulations of the Helsinki Declaration (2013) and approved by the Ethics Committee of the Privolzhsky Research Medical University. Informed consent forms were signed by all patients.

Instrumentation. Cryo-destruction of skin melanomas was performed by means of an original cryo-instrument cooled by the continuous flow of liquid nitrogen through a circular channel (Figures 1,2) with replaceable applicators with the ring-like surface (of different diameters and different widths of the ring); a hard vertical tube was mounted over the ring [18].

Cryo-laser excision of skin melanoma [19] was performed as follows. The uncooled applicator was placed on the preliminary moistened skin around the tumor so that the area was covered by the tube opening. Then, the coolant began circulating through the supply pipe, the circular cavity, and the exit pipe; with that in place the working surface was kept cool during the procedure. The instrument became firmly attached to the target skin area due to the adhesion between the applicator and the underlying skin. After that, liquid nitrogen was poured into the main tube, irrigating the tumor and nearby tissues. After $1.5 \mathrm{~min}$ of exposure, the temperature of the skin layers under the tumor reached $-40^{\circ} \mathrm{C}$ (as measured by a thermocouple). The proposed cryo-instrument provides for deep cryo-destruction, flexible cryo-exposure, and reduced traumatization by avoiding mechanical damage to the tumor tissue. Together, the applicator and cryo-irrigation reduce the time needed for complete freezing.

For a radical cryo-destruction of skin melanoma, 3-4 freezing cycles are performed (depending on the size of the neoplasia) with complete spontaneous thawing; then, the entire frozen block (tumor + surrounding tissue) is excised with a cryo-laser. Radiation from the $\mathrm{CO}_{2}$ laser is focused on the frozen tissue adjacent to the applicator, mainly along the $-20^{\circ} \mathrm{C}$ isotherm. On the line of the laser cut, an additional cryo-exposure is carried out by the Ledok cryo-instrument (developed

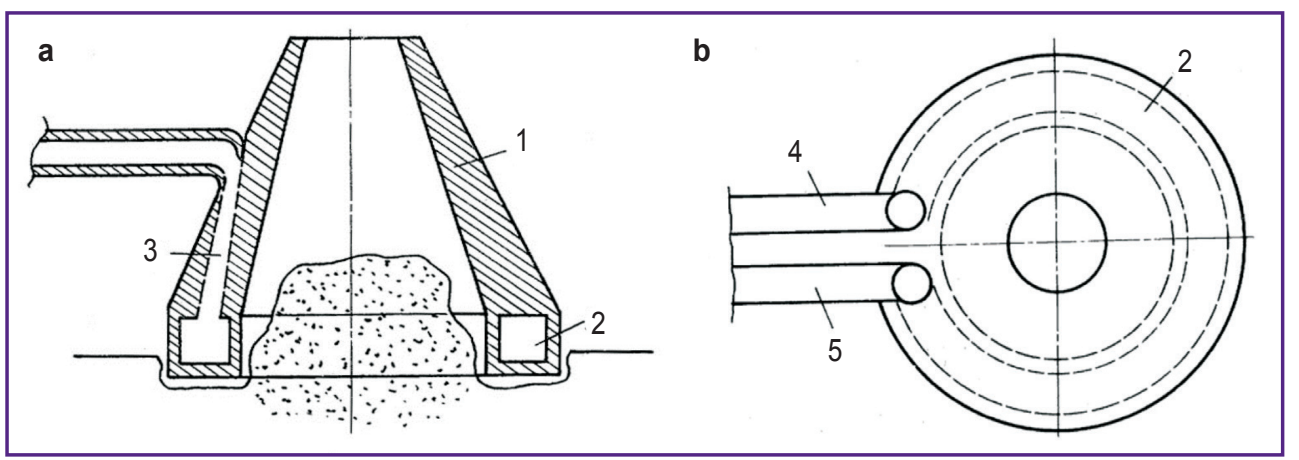

Figure 1. Schematic representation of the cryosurgical instrument:

(a) front view: 1 - body, 2 - circular cavity, 3- liquid nitrogen pipe; (b) top view: 2 - circular cavity, 4 - coolant entry pipe, 5 - coolant exit pipe

Figure 2. Surface-spreading melanoma $\mathrm{T}_{3 \mathrm{a}} \mathrm{N}_{0} \mathrm{M}_{0}$ (IIA stage according to AJCC):

(a) before cryotherapy; (b) after cryotherapy
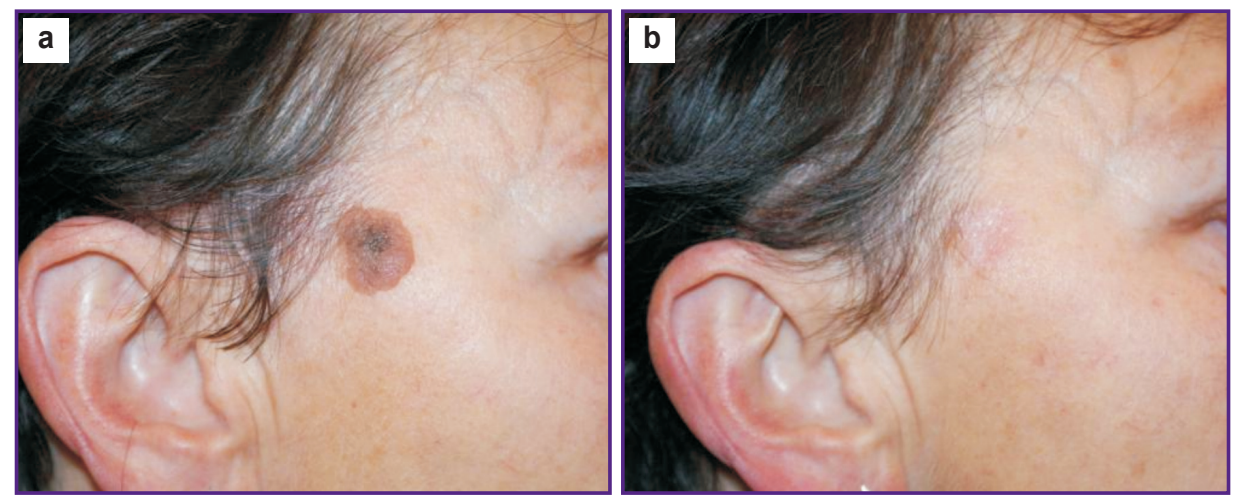
by Dr. V.I. Kochenov, Nizhny Novgorod, Russia). Such an excision can also be performed with the help of the EHVC-500 electrosurgical instrument - electro-knife (Elmed, Russia). Control biopsy is taken from the edges and the bottom of the postoperative wound, as well as along its circumference at distances of 0.5 to $2.5 \mathrm{~cm}$ from the wound boundaries (depending on the thickness of the tumor). The postoperative wound is sutured layer by layer; a temporary drainage catheter is left in the wound (see Figure 2).

The deep cooling with the adhesion allows the surgeon to freeze the melanoma and block its blood supply simultaneously from all sides, without mechanically touching the tumor itself. The vertical hard tube accelerates the process of freezing by simultaneously and directly exposing the exophytic part of the tumor to liquid nitrogen. The tube opening can be used for taking cryo-biopsy samples from the tumor without removing the instrument from the frozen tissue.

Evaluation of the immune status. To determine the immune-stimulating therapeutic effect, the immunological status was examined before the treatment, after it, and every 2 months during the first year and every 3 months during the years 2 to 5 . The very first test for the cellular immunity is carried out at the time when the diagnosis of skin melanoma is suspected. In the case melanoma is ruled out, no further monitoring of this patient takes place. In patients with confirmed melanoma, the patient's immune status is determined before and after surgery; the humoral and cellular immunity is evaluated with the help of monoclonal antibodies to lymphocyte subpopulations (including cytotoxic ones) - CD3, CD16, CD4, CD8, and CD4/CD8. To evaluate the immune status we used the immune regulation index (IRI) - the ratio of $\mathrm{T}$ helpers to $\mathrm{T}$ cytotoxic cells (CD4/CD8).

Data processing. To assess the normality of the data distribution, the Shapiro-Wilk criterion was used; for the comparison, the Student's t-criterion for related populations was used. Statistical significance was accepted at $p \leq 0.05$.

\section{Results}

In 126 patients with diagnosed skin melanoma, the immune status was examined before the treatment, after it, and every 2 months during the first year and every 3 months during the years 2 to 5 . Humoral and cellular immunity was assessed using monoclonal antibodies to CD3, CD6, CD4, CD8, and CD4/CD8, CD72 (Table 1). Prior to the cryosurgical treatment, the levels of CD4, CD8, and CD72 were below normal limits.

In Table 2, the CD4/CD8 ratio (IRI) in patients with different forms of skin melanoma after the treatment is shown.

Most melanomas in this group of patients were of the epithelioid type: 68 of 126 patients (53.9\%). In these patients with either early superficial neoplasia
Table 1

Immunological profile of patients with skin melanoma before and after cryosurgical treatment $(\mathrm{M} \pm \mathrm{m})$

\begin{tabular}{|c|c|c|c|c|}
\hline \multirow{2}{*}{ Parameter } & \multirow{2}{*}{ Units } & \multirow{2}{*}{$\begin{array}{l}\text { Normal } \\
\text { values }\end{array}$} & \multicolumn{2}{|c|}{ Patients ( $n=126$ ) } \\
\hline & & & Before treatment & After treatment \\
\hline \multirow{2}{*}{ CD3 } & $\%$ & $60-76$ & $67.1 \pm 2.7$ & $67.2 \pm 2.6$ \\
\hline & $\times 10^{6} \mathrm{~g} / \mathrm{L}$ & $1100-1700$ & $1251 \pm 228$ & $1079 \pm 79$ \\
\hline \multirow{2}{*}{ CD4 } & $\%$ & $38-46$ & $37.1 \pm 2.4$ & $42.5 \pm 2.2$ \\
\hline & $\times 10^{6} \mathrm{~g} / \mathrm{L}$ & $700-1100$ & $\downarrow 646 \pm 45$ & $759 \pm 50$ \\
\hline \multirow{2}{*}{ CD8 } & $\%$ & $31-40$ & $27.0 \pm 1.3$ & $29.6 \pm 3.8$ \\
\hline & $\times 10^{6} \mathrm{~g} / \mathrm{L}$ & $500-1000$ & $\downarrow 424 \pm 30$ & $537 \pm 40^{*}$ \\
\hline $\mathrm{CD} 4 / \mathrm{CD} 8$ & arb. units & $1.2-2.0$ & $0.6 \pm 0.1$ & $1.5 \pm 0.2^{* *}$ \\
\hline \multirow{2}{*}{ CD16 } & $\%$ & $10-19$ & $10.9 \pm 1.8$ & $14.2 \pm 2.1$ \\
\hline & $\times 10^{6} \mathrm{~g} / \mathrm{L}$ & $200-400$ & $180 \pm 32$ & $303 \pm 60$ \\
\hline \multirow{2}{*}{ CD72 } & $\%$ & $11-16$ & $\downarrow 8.5 \pm 1.1$ & $10.7 \pm 0.8$ \\
\hline & $\times 10^{6} \mathrm{~g} / \mathrm{L}$ & $200-400$ & $166 \pm 14$ & $207 \pm 23$ \\
\hline
\end{tabular}

Note. Significance of the differences when compared with the "before treatment" values: ${ }^{*} p<0.05 ;{ }^{* *} p<0.01$.

Table 2

Immune regulatory index in patients with different forms of skin melanoma after treatment

\begin{tabular}{lcc}
\hline \multicolumn{1}{c}{ Type of melanoma } & $\begin{array}{c}\text { Number of patients } \\
(\mathrm{n}=126)\end{array}$ & CD4/CD8 \\
\hline $\begin{array}{l}\text { Superficial } \\
\text { Nodular }\end{array}$ & 54 & $0.85 \pm 0.25$ \\
\hline $\begin{array}{l}\text { Ulcerated invasive } \\
\text { with inflammation (hyperemia) }\end{array}$ & 42 & $0.5 \pm 0.2$ \\
\hline Achromatic (red, pink) & 24 & $2.5 \pm 0.3$ \\
\hline None & 6 & $2.8 \pm 0.1$ \\
\hline
\end{tabular}

or ulcerated nodal forms, the CD4/CD8 ratio before treatment was $0.6 \pm 0.1$. The cryosurgical treatment of skin melanoma resulted in a 2-fold increase in the CD4/ CD8; after 6 months, this value increased even further to $1.5 \pm 0.2$. These data indicate that cryosurgical treatment had a stimulating effect on the immune system. The therapeutic consolidation lasted for 48 months (average) and depended on the reactivity of the immune system.

In 93 out of 96 patients who were followed up for a long time after this cryogenic treatment, the normal values of CD4/CD8 persisted for about 48 months. In 1 patient (82 years old), the CD4/CD8 value after circular cryo-excision did not change and was at the level of 0.6 arb. units. In the follow-up examination 4 years later, there were no signs of disease progression, and the CD4/CD8 ratio remained $\sim 0.5$ arb. units.

In the group of patients with melanoma of grade I-III (by Clark), who were followed up for a period from 12 
to 69 months, the only case of disease recurrence in the operation zone was in a 71-year-old woman with a disintegrating ear tumor of $5.0 \times 4.0 \times 3.0 \mathrm{~cm}$ in size. The local relapse and metastases to lymph cervical nodes were detected 8 months after the surgery. Death occurred 2 months later following the disease progression.

The five-year survival rate in patients after circular cryo-excision of melanoma with the Clark grade of IV-V was $80.0 \pm 8.9 \%$.

\section{Discussion}

The present study implies that the proposed circular cryo-excision of skin melanoma does not require a preceding radiation exposure, thus easing the management of the elderly and severely ill patients. For the first time, a significant immune stimulation by a cryoexposure was demonstrated in human subjects, with a therapeutic consolidation lasting for about 48 months. The use of cryosurgical treatment in patients with skin melanoma (including both cryo-fixation and cry-excision of the tumor) makes it possible to reduce 2-3-fold the volume of surrounding tissues to be removed, as compared with the traditional surgery.

The obtained results show that the circular cryoexcision of melanoma can be successfully applied to almost any tumor, especially in limited access locations. Reducing the volume of surgery did not cause any negative impact on the immediate and long-term results of the treatment; moreover, the immunological indices demonstrated an increase in the humoral and cellular immune response. In most cases, we found no contraindications against using this procedure; no preoperative procedures or general anesthesia is needed; the volume of removed tissues is reduced and thus, the cosmetic outcome improved. No postoperative complications were found in this study, which might be due to the ablastic reactions developing immediately after freezing (and during thawing): e.g. a local edema with increased interstitial pressure, lymphostasis, and thrombosis.

Cryogenic exposure (cryo-destruction and cryoextirpation) in patients with skin melanomas is usually well tolerated even by the elderly and by those having concomitant diseases. The obtained data support the use of this method in the treatment of primary foci of skin melanoma.

\section{Conclusion}

The proposed treatment of skin melanoma with the help of circular cryo-excision results in immunostimulation and consolidation (the latter lasts for $\sim 48$ months). The present study further rationalizes the use of this modality in surgical treatment of melanoma.

Research funding and conflict of interest. The study was not funded by any sources, and there are no conflicts of interest related to this study.

\section{References}

1. Malyshev A.S., Prokhorenkov V.I., Arutiunian G.A., Karacheva lu.V. The experience with diagnostics of melanocytic neoplasms with the use of epiluminescence microscopy: comparative characteristic of dermatoscopic algorithms. Klinicheskaya dermatologiya i venerologiya 2011; 1: 64-67.

2. Kotova T.G., Kochenov V.I., Tsybusov S.N., Gurin A.V. Ways to improve active detection of malignant neoplasms. Uspekhi sovremennoy nauki 2016; 1(4): 117-122.

3. Lemekhov V.G. Epidemiology, risk factors, screening of skin melanoma. Prakticheskaya onkologiya 2001; 8(4): 3-11.

4. Chu K.F., Dupuy D.E. Thermal ablation of tumours: biological mechanisms and advances in therapy. Nat Rev Cancer 2014; 14(3): 199-208, https://doi.org/10.1038/ nrc3672.

5. Ho T., Byrne P.J. Evaluation and initial management of the patient with facial skin cancer. Facial Plast Surg Clin North Am 2009; 17(3): 301-307, https://doi.org/10.1016/j. fsc.2009.04.002.

6. Kuflik E.G. Cryosurgery updated. J Am Acad Dermatol 1994; 31(6): 925-944, https://doi.org/10.1016/s01909622(94)70261-6.

7. Peksayar G., Altan-Yaycioglu R., Onal S. Excision and cryosurgery in the treatment of conjunctival malignant epithelial tumours. Eye 2003; 17(2): 228-232, https://doi.org/10.1038/ sj.eye.6700331.

8. Anandajeya W.V., Corrêa Z.M., Augsburger J.J. Primary acquired melanosis with atypia treated with mitomycin C. Int Ophthalmol 2009; 29(4): 285-288, https://doi.org/10.1007/ s10792-008-9225-3.

9. Erinjeri J.P., Clark T.W.I. Cryoablation: mechanism of action and devices. J Vasc Interv Radiol 2010; 21(8): S187S191, https://doi.org/10.1016/j.jvir.2009.12.403.

10. Sabel M.S. Cryo-immunology: a review of the literature and proposed mechanisms for stimulatory versus suppressive immune responses. Cryobiology 2009; 58(1): 1-11, https://doi. org/10.1016/j.cryobiol.2008.10.126.

11. Kotova T.G., Tsybusov S.N., Kochenov V.I., Tcyganov M.I. Application of cryogenic methods in skin diseases of different etiology. Dermatologic Surgery and Procedures 2018, https://doi.org/10.5772/intechopen.70509.

12. Rivas-Tolosa N., Ortiz-Brugués A., Toledo-Pastrana T., Baradad M., Traves V., Soriano V., Sanmartín V., Requena C., Martí R., Nagore E. Local cryosurgery and imiquimod: a successful combination for the treatment of locoregional cutaneous metastasis of melanoma: a case series. $J$ Dermatol 2016; 43(5): 553-556, https://doi.org/10.1111/13468138.13197.

13. Redondo P., del Olmo J., López-Diaz de Cerio A., Inoges S., Marquina M., Melero I., Bendandi M. Imiquimod enhances the systemic immunity attained by local cryosurgery destruction of melanoma lesions. J Invest Dermatol 2007; 127(7): 1673-1680, https://doi.org/10.1038/sj.jid.5700777.

14. de Moraes A.M., Pavarin L.B., Herreros F., de Aguiar Michelman F., Velho P.E., de Souza E.M. Cryosurgical treatment of lentigomaligna. J Dtsch Dermatol Ges 2007; 5(6): 477-480, https://doi.org/10.1111/j.1610-0387.2007.06331.x. 
15. Bub J.L., Berg D., Slee A., Odland P.B. Management of lentigo maligna and lentigo maligna melanoma with staged excision: a 5-year follow-up. Arch Dermatol 2004; 140(5): 552558, https://doi.org/10.1001/archderm.140.5.552.

16. Hill D.C., Gramp A.A. Surgical treatment of lentigo maligna and lentigo maligna melanoma. Australas $J$ Dermatol 1999; 40(1): 25-30, https://doi.org/10.1046/j.1440-0960. 1999.00311.x

17. Arlette J.P., Trotter M.J., Trotter T., Temple C.L.
Management of lentigo maligna and lentigo maligna melanoma: seminars in surgical oncology. J Surg Oncol 2004; 86(4): 179-186, https://doi.org/10.1002/jso.20081.

18. Shakhov V.Yu., Kochenov V.I., Rylkin A.I., Skachkov N.V., Drankin A.M. The tip of the cryosurgical equipment. USSR Author's Certificate SU 1124961 A. 1984.

19. Yakhontov N.E., Shakhov V.Yu., Skachkov N.V., Kochenov V.I., Terentev I.G. Method of excision of malignant neoplasms. USSR Author's Certificate SU 1790919 A1. 1993. 